\title{
Forwarding Redundancy in Opportunistic Mobile Networks: Investigation and Elimination
}

\author{
Wei Gao*, Qinghua $\mathrm{Li}^{\dagger}$, and Guohong $\mathrm{Cao}^{\ddagger}$ \\ ${ }^{*}$ Department of Electrical Engineering and Computer Science, The University of Tennessee, Knoxville \\ ${ }^{\dagger}$ Department of Computer Science and Computer Engineering, University of Arkansas \\ $\ddagger$ Department of Computer Science and Engineering, The Pennsylvania State University
}

\begin{abstract}
Opportunistic mobile networks consist of mobile devices which are intermittently connected via short-range radios. Forwarding in such networks relies on selecting relays to carry and deliver data to destinations upon opportunistic contacts. Due to the intermittent network connectivity, relays in current forwarding schemes are selected separately in a distributed manner. The contact capabilities of relays hence may overlap when they contact the same nodes and cause forwarding redundancy. This redundancy reduces the efficiency of resource utilization in the network, and may impair the forwarding performance if being ignored. In this paper, based on experimental investigations on the characteristics of forwarding redundancy in realistic mobile networks, we propose methods to eliminate unnecessary forwarding redundancy and ensure efficient utilization of network resources. We first develop techniques to eliminate forwarding redundancy with global network information, and then improve these techniques to be operable in a fully distributed manner with limited network information.
\end{abstract}

\section{INTRODUCTION}

Opportunistic Mobile Networks, also known as Delay/Disruption Tolerant Networks (DTNs) [7], consist of mobile devices which are connected only intermittently when they opportunistically contact each other, i.e., move into the communication range of their short-range radios (e.g., Bluetooth, WiFi). Such intermittent network connectivity can be a result of mobility, device sparsity or power outage. Examples include Vehicular Ad-hoc Networks (VANETs) [21] or groups of individuals moving in disaster recovery areas.

The intermittent network connectivity among mobile devices makes it difficult to maintain end-to-end communication links or global network information. To facilitate communication in opportunistic mobile networks, node mobility is exploited to let nodes physically carry messages as relays, which forward messages when they opportunistically contact other nodes. The key problem is hence how to make effective forwarding decisions, to ensure that the messages are carried by relays with the best chance to contact their destinations.

The opportunistic forwarding decision consists of two stages. First, the utility of a node for forwarding a message is determined. Due to the lack of global information about how to reach the destination, node's utility is evaluated by predicting the node's capability of contacting others in the future. Various utility functions evaluating the contact capability of nodes have been proposed based on node mobility patterns [20], stochastic node contact processes [15], [1], [10],

This work was supported in part by Network Science CTA under grant W911NF-09-2-0053. or social network concepts [13], [12]. Second, node utilities are applied to various forwarding strategies for different tradeoffs between forwarding performance and cost. Epidemic [19] and RAPID [1] optimize forwarding performance by utilizing all the nodes and contact opportunities for replicating messages. Most strategies only replicate messages to relays with high utilities and improve forwarding performance with lower cost.

In this paper, we envision that conventional wisdom has been focusing on developing various relay utility functions or forwarding strategies for opportunistic mobile networks, but generally ignore the large amount of forwarding redundancy produced by the current forwarding schemes, i.e., the calculated utility of a relay may not reflect its actual contribution on forwarding a message. The major reason for such redundancy is that the utility of a relay, in current forwarding schemes, is evaluated separately without considering the existence of other relays carrying replicas of the same message, and the contact capabilities of relays hence may overlap with each other. The relays may contact the same node at different times in the future, but only the first relay having contacted the destination delivers the message. The capabilities of all other relays contacting this node are redundant and wasted.

The existence of forwarding redundancy reduces the efficiency of resource utilization in opportunistic mobile networks, because some relays may have only little contribution on forwarding the message if the forwarding redundancy is neglected and inappropriately grows. Message replicas carried by these relays ineffectively consume the limited network resources including channel bandwidth and local storage, and further impair the performance of forwarding other messages.

The major focus of this paper, therefore, is to correctly identify and eliminate forwarding redundancy from relays' utilities, so as to improve the efficiency of resource utilization and forwarding performance in opportunistic mobile networks. Our basic idea is to develop cost-effective methods which identify the nodes that existing relays are likely to contact in the future, so that forwarding redundancy can be eliminated by avoiding these nodes from being contacted again, when the contact capabilities of other relays are later evaluated. More specifically, we made the following contributions:

- Investigation. We investigate characteristics of forwarding redundancy from both theoretical and experimental perspectives. We first formulate a theoretical framework to study the variations of forwarding performance and redundancy when message replicas are created, and then conduct experimental studies using existing forwarding 
schemes over real-world mobile network traces. We observe that some message replicas contribute little on improving the delivery ratio, and up to $70 \%$ of relays' utilities in current forwarding schemes are redundant. This result seriously impairs forwarding effectiveness and highlights the necessity of redundancy elimination.

- Elimination. The major challenge of eliminating forwarding redundancy is the lack of global network information. This makes it hard to estimate the cumulative contact capability of existing relays and determine forwarding redundancy. To address this challenge, we first propose a scheme to eliminate forwarding redundancy with global network information, and then make it distributed. We provide formal analysis on the accuracy of distributed redundancy elimination, and propose two alternative methods to correct the possible errors during redundancy elimination due to incompleteness of network information. After redundancy elimination, limited resources at each relay are effectively allocated to messages according to the relay's forwarding utilities. Evaluation results show that the forwarding performance after redundancy elimination is improved by $20 \%$ with $40 \%$ less cost.

The rest of this paper is organized as follows. Section II describes our network model and motivation of eliminating forwarding redundancy. Based on investigation results in Section III, our redundancy elimination schemes are described in Section IV. The performance of our proposed schemes is evaluated by trace-driven simulations in Section V. Section VI reviews related work and Section VII concludes the paper.

\section{OVERVIEW}

\section{A. Network Model and Assumptions}

Opportunistic contacts among mobile devices are described by network contact graph (NCG) $G(V, E)$, where contact process between nodes $i, j \in V$ is modeled as an edge $e_{i j} \in E$, and $e_{i j}$ only exists if $i$ and $j$ have contacted before.

We focus on minimizing the forwarding cost, measured by the average number of replicas created per message. We consider that each relay has only limited resources of channel bandwidth and local buffer. When multiple messages are forwarded to the same relay, their priorities are determined to maximize the effectiveness of utilizing the relay's resources. We assume a well-defined communication mechanism at and below the link layer, and the consideration of link quality or channel interference is beyond the scope of this paper.

We assume that each message has a finite lifetime $T$. Letting the forwarding delay be a random variable $X \in(0,+\infty]$, the expected delay is measured as $\mathbb{E}\{X \mid X \leq T\}$ and the delivery ratio is $\mathbb{P}(X \leq T)$. Since the delivery ratio and expected delay are correlated and increase simultaneously when $T$ increases, in this paper we measure forwarding performance using delivery ratio and will not evaluate delay separately.

\section{B. Motivation}

Forwarding redundancy in opportunistic mobile networks is illustrated by the example in Figure 1. Figure 1(a) only shows part of NCG near the data source $S$, and none of the

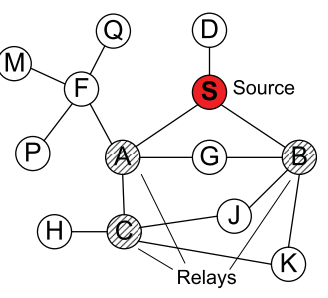

(a) Network contact graph

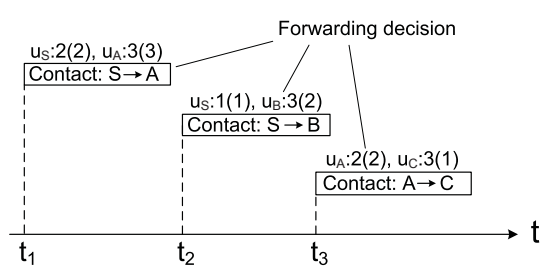

(b) Forwarding decisions
Fig. 1. Illustration of forwarding redundancy. Utility $u_{i}$ indicates the number of nodes that $i$ contacts, and the value within brackets is the value of $u_{i}$ after redundancy elimination. Message is replicated from $i$ to $j$ if $u_{i}<u_{j}$.

nodes in Figure 1(a) has contacted the destination, which is far away and not shown in the figure. In this case, the node's capability of contacting other nodes is used as its utility for forwarding decision ${ }^{1}$, and forwarding redundancy occurs when the relays $A, B$ and $C$ contact many nodes in common. In particular, when $B$ receives message replica at time $t_{2}$ from $S$ and becomes a relay, node $G$ contacted by $B$ is also contacted by another existing relay $A$. Hence, the contact of $B$ to $G$ is redundant. $B$ 's utility without such redundancy, measured in number of nodes that $B$ has contacted, should be 2 instead of 3. Similarly, contacts of relay $C$ to nodes $J$ and $K$ are redundant because of the existence of $B$, and this redundancy reduces $C$ 's utility from 3 to 1 .

This redundancy would impair the forwarding effectiveness. For example, with the existence of $B$, replicating message from $A$ to $C$ at time $t_{3}$ is ineffective because it only increases the cumulative number of nodes that the relays contact by $(6-5) / 5=20 \%$, but increases the number of message replicas by $33 \%$. $C$ may also prevent node $F$ with higher utility from being used as relay when forwarding strategy like Delegation [6] is used, due to the "fake" high utility of $C$.

Elimination of this redundancy is challenging due to the lack of global network information. When $S$ replicates message to $B$ at time $t_{2}, A$ may not know the existence of relay $B$ if $A$ has disconnected with $S$. Hence, when $A$ determines whether to replicate message to $C$ at time $t_{3}$, the redundancy between $B$ and $C$ on nodes $J$ and $K$ is hard to be eliminated.

\section{Metrics}

We measure forwarding redundancy as follows:

Definition 1: Redundancy percentage $P_{k}\left(t_{1}, t_{2}\right)$ of $k$ existing relays during time period $\left[t_{1}, t_{2}\right]$ is defined as

$$
P_{k}\left(t_{1}, t_{2}\right)=1-\left|\bigcup_{i=1}^{k} \mathbb{N}_{i}\left(t_{1}, t_{2}\right)\right| / \sum_{i=1}^{k}\left|\mathbb{N}_{i}\left(t_{1}, t_{2}\right)\right|,
$$

where node $j \in \mathbb{N}_{i}\left(t_{1}, t_{2}\right)$ if it is contacted by the $i$-th relay during time period $\left[t_{1}, t_{2}\right]$. The $i$-th relay belongs to $\mathbb{N}_{i}\left(t_{1}, t_{2}\right)$.

Forwarding redundancy varies when different utility functions are used for forwarding decision. In general, the utility of node $i$ is calculated as $U_{i}=\sum_{j=1}^{N} c_{i j}$, where $N$ is number of nodes in the network and $c_{i j}$ is the capability of node $i$ contacting $j$. Utility functions are classified into two categories according to the network information used to measure $c_{i j}$.

\footnotetext{
${ }^{1}$ Nodes $i$ and $j$ are not included in calculating their utilities when $i$ decides whether to replicate message to $j$. Existing relays are not included either.
} 
The first category is observational utilities, in which $c_{i j}$ is measured by direct network observations in the past. These observations include pairwise contact frequency (Freq) [5] and elapsed time since last contact (ElapsedTime) [3]. Betweenness [8] is also used in social-aware forwarding schemes [13], and defines $c_{i j}=\sum_{k=1}^{j-1} \frac{g_{j k}(i)}{g_{j k}}$, where $g_{j k}$ is the number of shortest paths between node $j$ and $k$ on the NCG and $g_{j k}(i)$ is the number of such paths passing node $i$. Betweenness hence indicates the relative importance of node $i$ in facilitating communication among other nodes.

The second category is probabilistic utilities, where $c_{i j}$ indicates pairwise contact probability derived from node contact process. PROPHET [15] increases $c_{i j}$ by $\left(1-c_{i j}\right) \cdot p_{i n i t}$ each time when $i$ and $j$ contact, and CCP [12] defines $c_{i j}=1-e^{-\lambda_{i j} T}$ where $\lambda_{i j}$ is the pairwise contact rate.

These utilities apply to various forwarding strategies. In Compare-and-Forward [3], [5], a relay replicates messages to nodes with higher utility than itself. Delegation [6] reduces the number of replicas, such that a relay only replicates message to nodes with higher utility than any existing relay that it is aware of. In Spray-and-Focus [18], the maximum number of message replicas is fixed and a relay forwards message to another node without retaining a local copy.

In this paper, we study forwarding redundancy over various combinations of forwarding strategies and utility functions. Note that there are more forwarding strategies and utility functions having been developed than the ones mentioned above. Our goal is not to address forwarding redundancy for each of them, but to demonstrate the general impact of this redundancy on forwarding performance, as well as the universal methodology for practical redundancy elimination.

\section{Traces}

Four sets of opportunistic mobile network traces are used in this paper. They record contacts among mobile devices with Bluetooth or WiFi interfaces moving in suburban areas (DieselNet [1]), conference site (Infocom [13]) and university campus (MIT Reality [4], UCSD [17]). Bluetooth-enabled devices periodically detect their peers nearby, and a contact is recorded when two devices move close. WiFi-enabled devices search for nearby WiFi Access Points (APs) and associate themselves to the APs with the best signal strength. A contact is recorded when two devices are associated to the same AP. As summarized in Table I, the four traces differ in their contact type, network scale and node contact frequency.

\section{INVESTIGATION}

In this section, we investigate the characteristics of forwarding redundancy from both theoretical and experimental aspects. We first provide theoretical insights on variations of delivery ratio and redundancy percentage when the message is being replicated, and then investigate these variations on realworld traces listed in Table I. Our findings are summarized as follows, and generally highlight the necessity of eliminating forwarding redundancy in opportunistic mobile networks.

- Forwarding redundancy exists in current schemes and seriously impairs the forwarding effectiveness.
- Message delivery ratio and redundancy percentage are closely correlated.

- The practical variations of delivery ratio and redundancy percentage accurately match our theoretical expectations.

TABLE I

TRACE SUMMARY

\begin{tabular}{c|cccc}
\hline Trace & DieselNet & Infocom & MIT Reality & UCSD \\
\hline \hline Network type & WiFi & Bluetooth & Bluetooth & WiFi \\
Contact type & Direct & Direct & Direct & AP-based \\
No. devices & 40 & 78 & 97 & 275 \\
Duration (days) & 20 & 4 & 246 & 77 \\
No. contacts & 3,268 & 182,951 & 114,046 & 123,225 \\
$\begin{array}{c}\text { No. contacts } \\
\text { per pair per day }\end{array}$ & 0.102 & 7.52 & 0.049 & 0.021 \\
\hline
\end{tabular}

\section{A. Theoretical Framework}

1) Delivery Ratio: We assume that a message is generated at time $t_{0}$ and expires at time $t_{e}$, with lifetime $T=t_{e}-t_{0}$. As a result, the message delivery ratio ${ }^{2}$ with $k$ relays is

$$
D_{k}\left(t_{0}, t_{e}\right)=1-\prod_{i=1}^{k}\left(1-\frac{n_{i}}{N}\right),
$$

where $N$ is the number of nodes in the network, $n_{i}=$ $\left|\mathbb{N}_{i}\left(t_{i}, t_{e}\right)\right|$ and $t_{i}$ is the time when the message is replicated to the $i$-th relay. When message is replicated to another relay $R_{k+1}, D_{k}$ increases by $\Delta D_{k}=\left(1-D_{k}\right) \cdot \frac{n_{k+1}}{N}$. If $n_{i}$ are i.i.d. stationary random variables with $\mathbb{E}\left\{n_{i}\right\}=\mu_{c}$, we have

$$
\mathbb{E}\left\{D_{k}\right\}=1-\left(1-\mu_{c} / N\right)^{k} \text {. }
$$

In Eq. (2), both the destination and the nodes in $\mathbb{N}_{i}$ are assumed to be uniformly distributed in the network. In social computing applications with community structures, such distribution may be highly skewed. Relays within the same community may contact the same nodes and lead to lower delivery ratio. In contrast, $\mathbb{N}_{i}$ of relays within different communities may not overlap at all.

Comparatively, delivery ratio without considering forwarding redundancy is $\widetilde{D}_{k}=\frac{1}{N} \cdot \sum_{i=1}^{k} n_{i}$ with $\sum_{i=1}^{k} n_{i} \leq N$, and $\mathbb{E}\left\{\widetilde{D}_{k}\right\}=k \mu_{c} / N$. Figure 2(a) illustrates the difference between $\mathbb{E}\left\{D_{k}\right\}$ and $\mathbb{E}\left\{\widetilde{D}_{k}\right\}$ when $k$ increases. While $\mathbb{E}\left\{\widetilde{D}_{k}\right\}$ linearly increases with $k, \mathbb{E}\left\{D_{k}\right\}$ increases more slowly. This difference is larger when $k$ increases or $\mu_{c}$ is smaller. Since the distribution of node contact capability is highly skewed in reality [13], value of $\mu_{c}$ is low and the actual delivery ratio is much lower than that indicated by relays' utilities.

2) Redundancy Percentage: The redundancy percentage of $k$ relays is $P_{k}=1-N \cdot D_{k} / N_{k}$, where $D_{k}$ is defined in Eq. (2) and $N_{k}=\sum_{i=1}^{k} n_{i}$. Since $\mathbb{E}\left\{\frac{1}{N_{k}}\right\} \geq \frac{1}{k \mu_{c}}$, we have

$$
\mathbb{E}\left\{P_{k}\right\} \leq 1-N \cdot \mathbb{E}\left\{D_{k}\right\} /\left(k \mu_{c}\right),
$$

and this upper bound is asymptotically tight because $\mathbb{E}\left\{P_{N}\right\}=$ $1-N \cdot \mathbb{E}\left\{D_{N}\right\} /\left(N \mu_{c}\right)$ when $N$ is sufficiently large. When a message is replicated to another relay $R_{k+1}, P_{k}$ increases by

$$
\begin{aligned}
\Delta P_{k} & =P_{k+1}-P_{k}=\frac{N \cdot D_{k}}{N_{k}}-\frac{N \cdot D_{k+1}}{N_{k}+n_{k+1}} \\
& =\frac{N \cdot n_{k+1}}{N_{k}\left(N_{k}+n_{k+1}\right)} \cdot\left(D_{k}-\frac{N_{k}}{N}\left(1-D_{k}\right)\right) .
\end{aligned}
$$

\footnotetext{
${ }^{2}$ The delivery ratio of a single message equals to its probability to be delivered to the destination before expiration.
} 

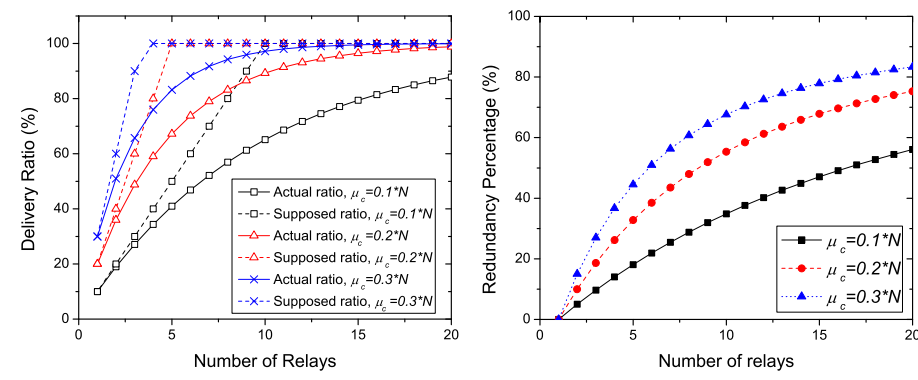

(a) Comparing $\mathbb{E}\left\{D_{k}\right\}$ and $\mathbb{E}\left\{\widetilde{D}_{k}\right\}$

(b) Redundancy percentage

Fig. 2. Theoretical variation of delivery ratio and redundancy percentage

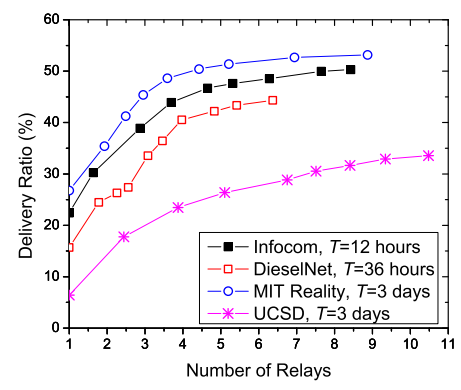

(a) Delivery ratio

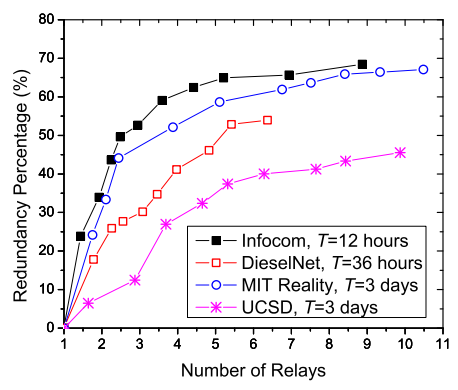

(b) Redundancy percentage
Fig. 3. Delivery ratio and redundancy percentage in different traces when a message is replicated to more relays

Theorem 1: For $\forall k \geq 1, \Delta P_{k} \geq 0$.

Proof: Consider functions $f\left(n_{1}, \ldots, n_{k}\right)=\frac{1}{N} \sum_{i=1}^{k} n_{i}=$ $N_{k} / N$ and $g\left(n_{1}, \ldots, n_{k}\right)=\frac{1-\prod_{i=1}^{k}\left(1-\frac{n_{i}}{N}\right)}{\prod_{i=1}^{k}\left(1-\frac{n_{i}}{N}\right)}=\frac{D_{k}}{1-D_{k}}$. For $\forall i \in[1, k]$ we have $\frac{\partial f}{\partial n_{i}}=\frac{1}{N}$ and $\frac{\partial g}{\partial n_{i}}=\frac{1}{N\left(1-\frac{n_{i}}{N}\right)} \cdot(1+$ $\left.g\left(n_{1}, \ldots, n_{k}\right)\right)$. Since $f(0, \ldots, 0)=g(0, \ldots, 0)=0$, we have $f\left(n_{1}, \ldots, n_{k}\right) \leq g\left(n_{1}, \ldots, n_{k}\right)$ for $\forall n_{1}, \ldots, n_{k} \in(0, N]$ and the theorem follows.

Theorem 1 shows that forwarding redundancy does not always decrease when a message is replicated, and this is also illustrated in Figure 2(b) by using its upper bound in Eq. (4). When $\mu_{c}$ increases, a node can be contacted by more relays and hence leading to higher redundancy percentage.

Moreover, replicating message to more relays may increase the relay coverage and redundancy percentage simultaneously. When the number of message replicas is small, each replica has limited forwarding redundancy but greatly increases the delivery ratio. In contrast, when a message is replicated to more relays, the newly created replicas become redundant and only contribute little to the forwarding performance.

\section{B. Trace Studies}

We investigate the characteristics of forwarding redundancy in the traces listed in Table I. In each experiment, a message is generated with random source and destination over 100 simulation runs. A warm-up period is reserved before the message is generated, for nodes to calculate their utilities.

1) Impact of forwarding redundancy: We first vary the number of message replicas using the Spray-and-Focus strategy [18] and the utility function of CCP [12]. Message lifetime

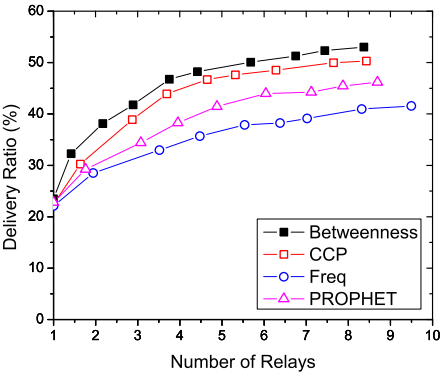

(a) Delivery ratio

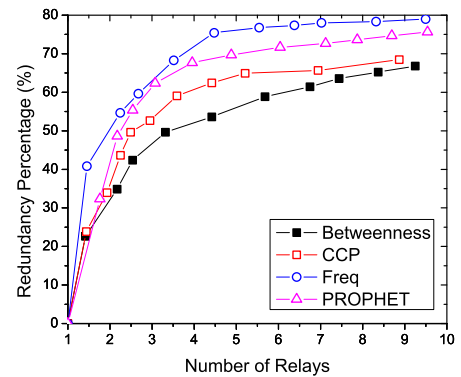

(b) Redundancy percentage
Fig. 4. Delivery ratio and redundancy percentage in the Infocom trace with different utility functions

TABLE II

CURVE FITTING ON DELIVERY RATIO

\begin{tabular}{c|ccc}
\hline Trace & $D_{\max }$ & $m$ & fitting error \\
\hline DieselNet & 0.5233 & 0.2728 & $1.65 \times 10^{-3}$ \\
Infocom & 0.5021 & 0.4182 & $1.307 \times 10^{-3}$ \\
MIT Reality & 0.5402 & 0.4549 & $1.077 \times 10^{-3}$ \\
UCSD & 0.4893 & 0.1206 & $2.48 \times 10^{-4}$ \\
\hline
\end{tabular}

$T$ is adaptively determined in different traces to ensure that the designated number of message replicas is created.

As shown in Figure 3, both delivery ratio and redundancy percentage increase when more message replicas are created. This increase is determined by the trace-dependent node contact patterns. In the MIT Reality trace, when the number of relays is smaller than 3 , the redundancy percentage is lower than $40 \%$, and each message replica improves the delivery ratio by $10 \%$. However, the other 6 message replicas being created later only improve delivery ratio by another $10 \%$, but increase redundancy percentage to $70 \%$. Similar cases are also found in other traces. These results show that inappropriate increase of forwarding redundancy only has little contribution on forwarding performance but impairs its effectiveness.

When more message replicas are created, the increase of delivery ratio shown in Figure 3(a) is consistent with our theoretical expectations in Section III-A. To validate this consistency, we perform least-square curve fitting in Figure 3(a) using formula $\mathbb{E}\left\{D_{k}\right\}=D_{\max } \cdot\left(1-(1-m)^{k}\right)$ where $m=\mu_{c} / N$, and the results in Table II show that the fitting error is lower than $2 \times 10^{-3}$ in all cases. Similar consistent results can be found in terms of redundancy percentage by comparing Figure 3(b) with Figure 2(b).

In Figure 4 we also investigate the impact of different utility functions. Freq and PROPHET lead to lower delivery ratio and higher redundancy because they inaccurately measure node contact capability. CCP and Betweenness perform better, especially when more message replicas are created.

2) Correlation Analysis: We are also interested in the correlation between delivery ratio and redundancy percentage. Figure 5 shows that the two metrics are closely correlated and both increase when message replicas are created. Note that there is an inflection point in each curve in Figure 5. Delivery ratio increases faster than redundancy percentage before the inflection point and vice versa. The position of the inflection point is trace-dependent, and also depends on the forwarding 


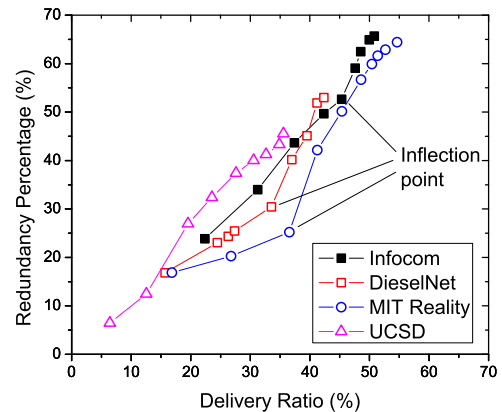

(a) Correlation in different traces

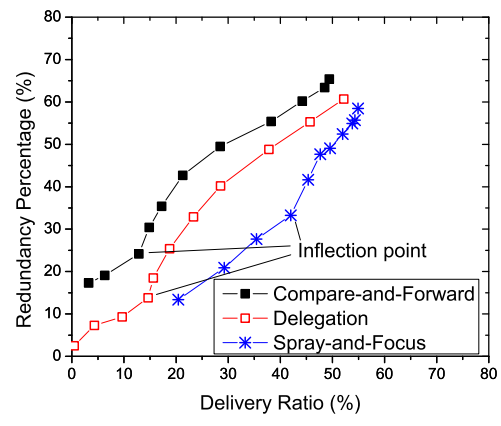

(b) Correlation with different forwarding strategies

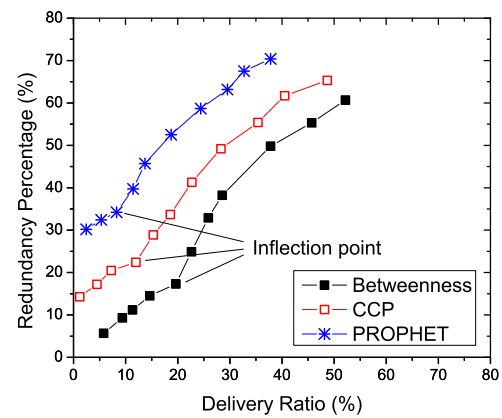

(c) Correlation with different utilities

Fig. 5. Correlation between delivery ratio and redundancy percentage. An inflection point is found in all cases.

strategy and utility function being used. Figure 5(b) shows that Compare-and-Forward is less effective and Spray-and-Focus performs better, when CCP is used as the utility function. Similarly, Figure 5(c) shows that PROPHET and CCP produce more redundancy than Betweenness does, with the Delegation forwarding strategy.

\section{ELIMINATION}

In this section, we eliminate the forwarding redundancy from the utilities used to evaluate relays' contact capabilities, so as to prevent this redundancy from affecting forwarding decisions and ensure efficient utilization of network resources.

Our basic idea is to maintain Cumulative Relay Information (CRI) for each message, which records the cumulative contact capability of relays to forward the message. The definition of CRI depends on the network information available at individual nodes and is described later. When a relay $R$ decides whether to replicate the message to node $A, R$ compares the contact capability of $A$ with the current CRI, and checks whether nodes contacted by $A$ have also been contacted by other relays. If so, this redundancy is eliminated from $A$ 's utility, which then reflects $A$ 's actual contribution on forwarding the message.

We first focus on eliminating forwarding redundancy with complete CRI at the global scope, and then extend this scheme to be distributed with incomplete CRI maintained at individual relays. Impact of this incompleteness to redundancy elimination is analyzed and addressed from various perspectives.

\section{A. Redundancy Elimination with Global CRI}

We first assume that each node knows the global CRI. In practice, this information can be provided from a backend server via $3 \mathrm{G}$ links. The global CRI maintains a quantity $C_{i}^{(k)}$ for each node $i$, which indicates the cumulative capability of the current $k$ relays contacting $i . C_{i}^{(0)}=0$ for $\forall i$. Each time when the message is replicated to another relay $R_{k+1}, C_{i}^{(k)}$ for each non-relay node $i$ is updated as

$$
C_{i}^{(k+1)}=f\left(C_{i}^{(k)}, c_{i}^{(k+1)}\right),
$$

where $c_{i}^{(k+1)}$ is the capability of $R_{k+1}$ contacting $i$, and is evaluated when the message is replicated to $R_{k+1} . f(\cdot)$ is a utility-dependent function with the following properties:

$$
\text { - Monotonicity. For } \forall k \in[1, N], C_{i}^{(k+1)} \geq C_{i}^{(k)} \text {. }
$$

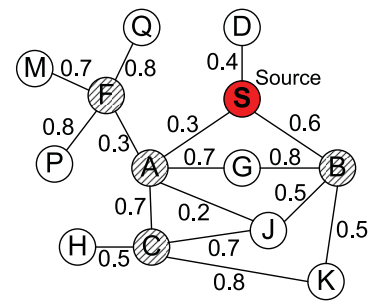

(a) Network contact graph. Numbers on the edges are the pairwise contact probabilities.

Fig. 6. The impact of redundancy elimination in Eq. (9) on forwarding decision with Delegation strategy.

- Convexity. For $\forall k \in[1, N], C_{i}^{(k+1)} \leq C_{i}^{(k)}+c_{i}^{(k+1)}$.

Forwarding redundancy caused by $R_{k+1}$ on node $i$ is identified as the difference between $C_{i}^{(k+1)}$ and $C_{i}^{(k)}+c_{i}^{(k+1)}$. Hence, after redundancy elimination, the utility of $R_{k+1}$ for forwarding this message is calculated as

$$
U_{k+1}=\sum_{i=1}^{N}\left(C_{i}^{(k+1)}-C_{i}^{(k)}\right)
$$

which indicates the actual contribution of $R_{k+1}$ on forwarding the message instead of $\sum_{i=1}^{N} c_{i}^{(k+1)}$. We design $f(\cdot)$ for observational and probabilistic utility functions, respectively.

1) Observational Utilities: For observational utilites including Freq, ElapsedTime and Betweenness, the contribution of $c_{i}^{(k+1)}$ to $C_{i}^{(k+1)}$ is reduced by the ratio between $c_{i}^{(k+1)}$ and $C_{i}^{(k)}+c_{i}^{(k+1)}$, i.e.,

$$
C_{i}^{(k+1)}=C_{i}^{(k)}+\frac{c_{i}^{(k+1)}}{C_{i}^{(k)}+c_{i}^{(k+1)}} \cdot c_{i}^{(k+1)} .
$$

Eq. (8) can be interpreted in various ways when being applied to different utility functions. For Freq, the chance for $R_{k+1}$ to provide useful contact capability to node $i$ is proportionally reduced due to the existing contact capability $C_{i}^{(k)}$. This reduction also applies to ElapsedTime, because the reciprocal of elapsed time since last contact equivalently measures contact frequency by assuming stationary contact process. For example, if two relays $A$ and $B$ contact node $i$ with the frequency 2 and 8 respectively, the cumulative $C_{i}^{(2)}=$ $2+\frac{8 \times 8}{2+8}=8.4$ according to Eq. (8). For the third relay $C$ contacting $i$ with the frequency $6, C_{i}^{(3)}=8.4+\frac{6 \times 6}{8.4+6}=10.9$.

For Betweenness, $c_{i}^{(k+1)}$ measures the number of nodes which can communicate with node $i$ via $R_{k+1}$. Betweenness 


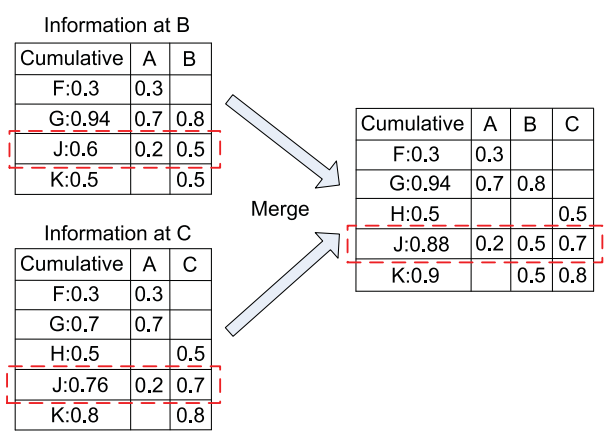

Fig. 7. Distributed maintenance of CRI based on the network in Figure 6 in opportunistic mobile networks is usually calculated in an ego-centric manner [8]. Forwarding redundancy exists when the neighborhood of $R_{k+1}$ on NCG overlap with that of other relays, and hence can be calculated similarly using Eq. (8).

2) Probabilistic Utilities: For probabilistic utilities including PROPHET and CCP, $c_{i}^{(k+1)}$ is the probability for $R_{k+1}$ to contact $i$. We assume that the contact process of each relay is independent, and $C_{i}^{(k+1)}=1-\prod_{j=1}^{k+1}\left(1-c_{i}^{(j)}\right)$. Hence,

$$
C_{i}^{(k+1)}=1-\left(1-C_{i}^{(k)}\right) \cdot\left(1-c_{i}^{(k+1)}\right) .
$$

The impact of redundancy elimination on forwarding decision is illustrated in Figure 6 . The utilities of $B$ and $C$ are reduced by $50 \%$ after their redundancy of contacting $G, J$ and $K$ is eliminated, and hence will not be used as relays. Instead, $A$ replicates message to $F$ which contacts more distinct nodes and has more contributions on forwarding the message.

\section{B. Distributed Elimination}

When the global CRI is unavailable, each relay maintains CRI in a distributed manner. Due to the lack of end-to-end network connectivity, the CRI maintained at relays may be incomplete and partially overlap with each other. For example, in the network shown in Figure 6, node $J$ is contacted by three relays $A, B$ and $C$. The CRI about node $J$ maintained at $B$ includes capability of $A$ and $B$ contacting $J$, but the CRI maintained at $C$ only includes that of $A$ and $C$.

Due to this possible overlapping, relays need to merge their maintained CRI when they contact, and the quantity $C_{i}^{(k)}$ is insufficient for maintaining CRI in a distributed manner. In the above example, it is difficult for relays $B$ and $C$ to correctly identify this overlapping and merge their CRI to calculate the cumulative capability of $A, B$ and $C$ contacting $J$.

In this case, CRI is maintained in a more fine-grained level. A relay maintains a list for each non-relay node $i$, and the list records the capability $c_{i}^{(j)}$ of each relay $R_{j}$ contacting $i$. When relays $B$ and $C$ in Figure 6 contact each other, their lists are merged to correctly calculate CRI of node $J$ based on Eqs. (8) or (9), and this process is illustrated in Figure 7.

The amount of storage space for maintaining CRI is related to the number of relays, which is much smaller than the number $(N)$ of nodes in the network. For example, the required space is $O\left(N \cdot(\log N)^{2}\right)$ when the Delegation strategy is used.

\section{Accuracy Analysis and Improvement}

When CRI is maintained in a distributed manner, accuracy of redundancy elimination may be impaired due to incompleteness of CRI. This incompleteness appears when a relay

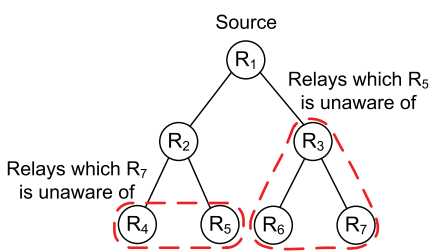

(a) Incomplete CRI at relays. Relays receive message replicas from their parents, and are indexed by their time receiving message replica.

Fig. 8. Incompleteness of CRI and its impact on forwarding decision. Delegation strategy is used.
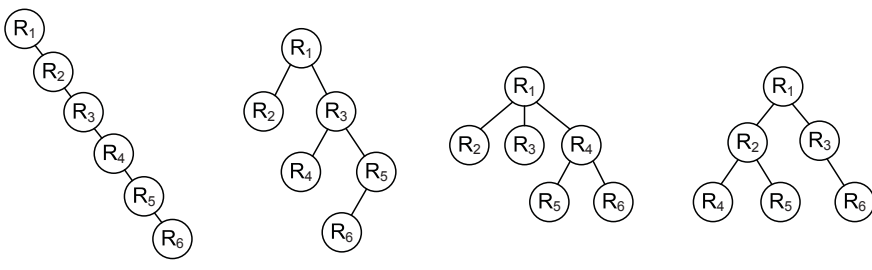

(a) $\left(N_{R}, K_{R}\right)=(1,1)$

(b) $\left(\mathrm{N}_{\mathrm{R}}, \mathrm{K}_{\mathrm{R}}\right)=(1,2)$

(c) $\left(\mathrm{N}_{\mathrm{R}}, \mathrm{K}_{\mathrm{R}}\right)=(1,3)$

(d) $\left(\mathrm{N}_{\mathrm{R}}, \mathrm{K}_{\mathrm{R}}\right)=(2,2)$

Fig. 9. MRTs with 6 relays and different combinations of $\left(N_{R}, K_{R}\right)$

is unaware of some other existing relays, and is illustrated in Figure 8(a) which describes forwarding process as a Message Replication Tree (MRT). Without loss of generality, we assume that communication links among $R_{1}, \ldots, R_{k-1}$ have been broken when the message is replicated to relay $R_{k}$. In Figure 8(a) when message is replicated from $R_{3}$ to $R_{7}, R_{7}$ knows $R_{2}$ because $R_{2}$ receives message replica earlier from $R_{1}$ which is also the parent of $R_{3}$ on MRT, but $R_{7}$ is unaware of the existence of $R_{4}$ and $R_{5}$ because the link between $R_{1}$ and $R_{2}$ has been broken. Similarly, neither $R_{4}$ nor $R_{5}$ knows $R_{3}, R_{6}$ and $R_{7}$.

Definition 2: The Blind Zone $(B Z) \mathbb{B}_{R_{i}}(t)$ of a relay $R_{i}$ at time $t$ is defined as a set of relays which receive message replicas before time $t$, such that a relay $R_{j} \in \mathbb{B}_{R_{i}}(t)$ if $R_{i}$ is unaware of the existence of $R_{j}$ at time $t$.

The BZs of relay $R_{5}$ and $R_{7}$ are indicated by dashed circles in Figure 8(a). Based on this definition, the incompleteness of CRI at time $t$ is measured by the average size of relays' BZ as $I_{k}=\frac{1}{k} \cdot \sum_{i=1}^{k}\left|\mathbb{B}_{R_{i}}(t)\right|$, where $k$ is the number of relays.

This incompleteness of CRI may cause false positive and false negative errors to forwarding decision. First, $R_{5}$ and $R_{7}$ in Figure 8 (b) contact node $A$ with probability 0.5 and 0.6 , and the actual utility of $R_{7}$ should be 2.2 instead of 2.5 according to Eq. (9). When Delegation strategy is used, $R_{7}$ should not receive message replica from $R_{3}$ because $R_{6}$ becomes relay earlier and $2.3>2.2$. Hence, $R_{7}$ is a false positive error. Second, $R_{3}$ incorrectly considers that $R_{7}$ has the highest utility of 2.5 among existing relays. This prevents $R_{8}$ from becoming a relay and leads to a false negative error.

We propose two alternative schemes to address these errors and improve the accuracy of redundancy elimination. First, we pre-regulate the forwarding process before a message is actually replicated to relays, so as to reduce the chance of errors. Second, we opportunistically adjust relays after they 


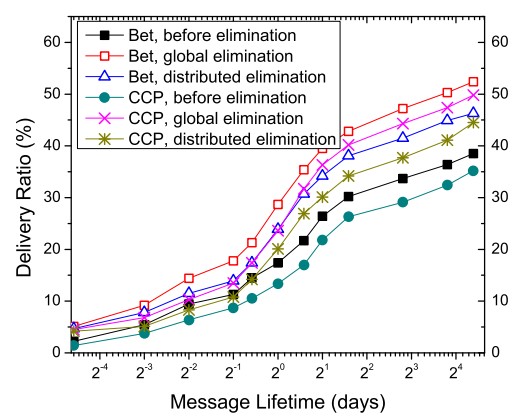

(a) Delivery ratio

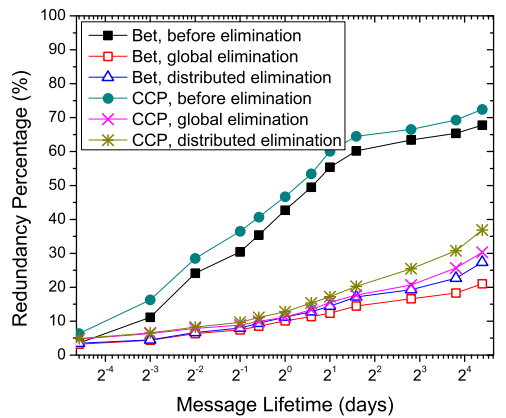

(b) Redundancy Percentage

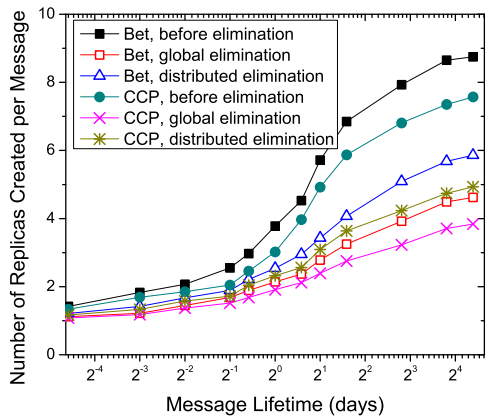

(c) Number of replicas created per message

Fig. 10. Performance of redundancy elimination with global and distributed CRI. Compare-and-Forward strategy is used in the MIT Reality trace. Our schemes eliminate forwarding redundancy by more than $50 \%$, and improve the cumulative delivery ratio by $20 \%$ with $40 \%$ less cost.

receive message replicas, when errors are detected.

1) Pre-regulation of Forwarding Process: A relay $R_{k}$ is inevitably blind to relays which receive message replica later and not from $R_{k}$ itself. Hence, we focus on ensuring that $R_{k}$ knows all the relays $R_{1}, \ldots, R_{k-1}$, i.e., $\left|\widetilde{\mathbb{B}}_{R_{k}}(t)\right|=\mid \mathbb{B}_{R_{k}}(t) \cap$ $\left\{R_{1}, \ldots, R_{k-1}\right\} \mid=0$ for $\forall t \geq T_{k}$, where $T_{k}$ is the time when $R_{k}$ receives the message replica. This is achieved by regulating forwarding process represented by MRT.

$\left|\widetilde{\mathbb{B}}_{R_{k}}(t)\right|$ is controlled by two parameters $N_{R}$ and $K_{R}$. First, $N_{R}$ is the maximum number of non-leaf relays at each level of MRT. Without loss of generality, we assume that the non-leaf relays at each level of MRT have larger indices than any leaf relay at the same level. Second, we only allow a non-leaf relay to replicate messages and produce at most $K_{R}$ new relays. MRTs with $\left(N_{R}, K_{R}\right)$ from $(1,1)$ to $(2,2)$ are illustrated in Figure 9.

Lemma 1: $\left|\widetilde{\mathbb{B}}_{R_{k}}(t)\right|=0$ for $\forall k \geq 1$ when $N_{R}=1$.

The proof of Lemma 1 can be found in Appendix. From Lemma 1, we immediately have the following theorem considering that $\left|\mathbb{B}_{R_{k-1}}(t)\right|=\left|\mathbb{B}_{R_{k}}(t)\right|=0$.

Theorem 2: When $N_{R}=1, I_{k}=\frac{(k-1)(k-2)}{2 k^{2}}$ for any $K_{R} \geq 1$.

$I_{k}$ increases with $K_{R}$ when $N_{R}>1$. Since the non-leaf relays usually have the best capability contacting others, we ensure that a sufficient number of message replicas is created.

2) Posterior Adjustment of Relays: The pre-regulation may prevent relays from receiving message replicas and affect the forwarding performance. Another way is to adjust the relays in a posterior manner, when the false positive and false negative errors are detected. These errors are detected when relays contact each other and update their maintained CRI.

A relay $R_{k}$ autonomously revokes itself by removing its message replica, when it detects false positive. For this detection, $R_{k}$ memorizes the situation at time $T_{k}$ when it received message replica. Each time when $R_{k}$ contacts another relay and updates its CRI, it recalculates its utility at time $T_{k}$. The false positive error is detected when $R_{k}$ realizes that it should not be a relay with the new utility. In Figure $8(\mathrm{~b}), R_{7}$ finds that its utility should be 2.2 instead of 2.5 when it contacts $R_{5}$, and realizes this is a false positive. After $R_{7}$ revoked itself, $R_{5}$ is responsible for notifying other relays to remove $R_{7}$ 's information from their maintained CRI.
Figure 8 (b) shows that a false negative error only happens after a false positive error. After relay $R_{7}$ revokes itself, the false negative error on $R_{8}$ is detected until $R_{3}$ or $R_{8}$ is notified about the revocation of $R_{7}$. Since $R_{3}$ may not be in contact with $R_{8}$ by then, $R_{3}$ spreads the information about this error among existing relays, so that $R_{8}$ receives message replica if it contacts any relay being aware of this false negative error.

The delay of error detection is determined by both the network scale and node contact pattern. Since the relays have good capabilities to contact other nodes, this delay is expected to be much shorter than the inter-contact time.

\section{Local Allocation of Relay Resources}

A relay has only limited local resources. When replicas of multiple messages are forwarded to a relay, its resources should be appropriately allocated. Such problem of local resource allocation has been studied in [1], but with the assumption that each message has equal size. Instead, we propose a generalized solution based on the relays' utilities after redundancy elimination.

When replicas of $M$ messages with sizes $s_{1}, \ldots, s_{M}$ are forwarded from relay $R_{1}$ to relay $R_{2}$ with buffer size $B$, the problem of resource allocation at $R_{2}$ is formulated as

$$
\max \sum_{k=1}^{M} U_{2}^{(k)} x_{k} \quad \text { s.t. } \sum_{k=1}^{M} s_{k} x_{k} \leq B,
$$

where $x_{k} \in\{0,1\}$ indicate whether the $k$-th message replica is forwarded to $R_{2}$, and $U_{2}^{(k)}$ is utility of $R_{2}$ defined in Eq. (7) for forwarding the $k$-th message. The forwarding redundancy between existing relays (including $R_{1}$ ) and $R_{2}$ has been eliminated from $U_{2}^{(k)}$. Eq. (10) ensures that $R_{2}$ 's resources are allocated to appropriate messages, such that $R_{2}$ has the most contributions on forwarding these messages. Since $B$ and $s_{k}$ in Eq. (10) are usually integers in numbers of bytes, Eq. (10) can be solved in pseudo-polynomial time $O(M \cdot B)$ using dynamic programming [16].

Due to the limited channel bandwidth and contact duration, $R_{1}$ may not be able to transmit all the message replicas selected by Eq. (10) to $R_{2}$ before the contact ends. The order for message replicas to be transmitted follows their order being selected when solving Eq. (10) using dynamic programming, due to the property of optimal substructure of Eq. (10). 


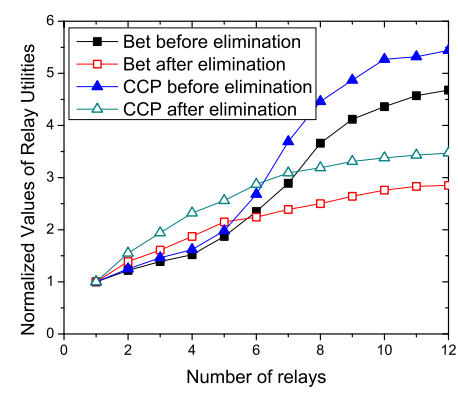

(a) Change of relays' utilities

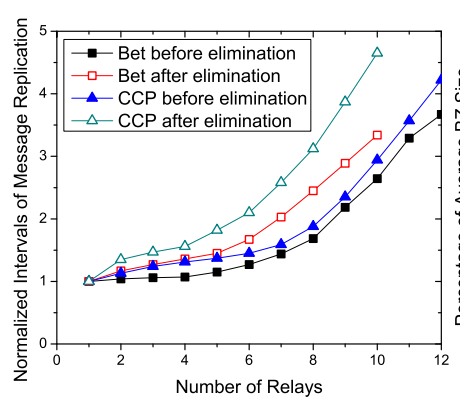

(b) Intervals of message replication

Fig. 11. Detailed effects of redundancy elimination

\section{Performance Evaluations}

In this section, we evaluate the performance of our schemes using the traces listed in Table I. The first half of each trace is used for nodes to collect network information and all the messages are generated at randomized times afterwards. We assume that the channel bandwidth is $1 \mathrm{Mbps}$ and the message size is uniformly distributed in $[10 M b, 50 M b]$. As reported in [9], most of contacts in the traces we use last long enough to transmit at least one message replica. The buffer size of nodes is uniformly distributed in $[50 M b, 500 M b]$ so that each node can carry at least one message replica.

\section{A. Performance of Redundancy Elimination}

We first evaluate the performance of redundancy elimination with global and distributed CRI. We generate a message every hour, and the evaluation results are shown in Figure 10, where Betweenness and CCP are used to represent observational and probabilistic utility functions, respectively.

Figure 10(b) shows that our schemes eliminate forwarding redundancy by over $50 \%$. This elimination enables effective resource utilization, leading to $20 \%$ improvement of delivery ratio and $40 \%$ forwarding cost reduction ${ }^{3}$. The contact capabilities of relays after redundancy elimination may still overlap, but this remaining redundancy is very limited compared to the relays' useful contact capabilities. Figure 10 also shows the impact of CRI incompleteness to redundancy elimination and forwarding performance. When CRI is maintained in a distributed manner, the CRI incompleteness increases redundancy percentage by $10 \%$, and forwarding cost by $20 \%$.

In Figure 11, we evaluate relays' utilities and intervals of message replication after redundancy elimination. For each message, the utility and replication interval of the first relay are used for normalization. The relays' utilities after redundancy elimination are reduced by $40 \%$, which is the major reason of the reduction in forwarding cost shown in Figure 10(c). It also takes longer time for each message to be replicated.

\section{B. Effectiveness of Accuracy Improvement}

The accuracy of redundancy elimination may be impaired due to CRI incompleteness at individual relays. Various techniques have been proposed in Section IV-C to correct

\footnotetext{
${ }^{3}$ We consider the overhead of maintaining CRI negligible because only small CRI metadata needs to be exchanged when relays contact.
}

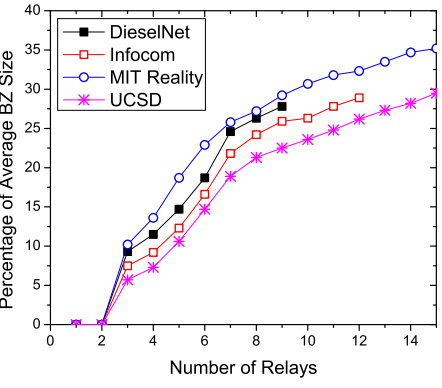

(a) BZ sizes in different traces

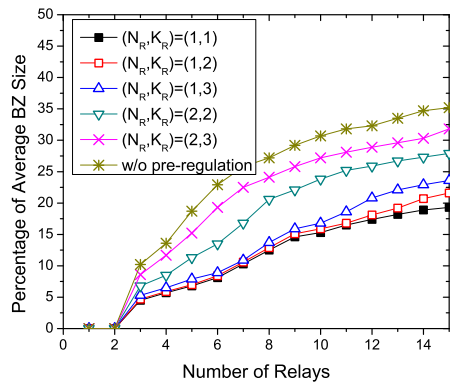

(b) BZ sizes in MIT Reality trace

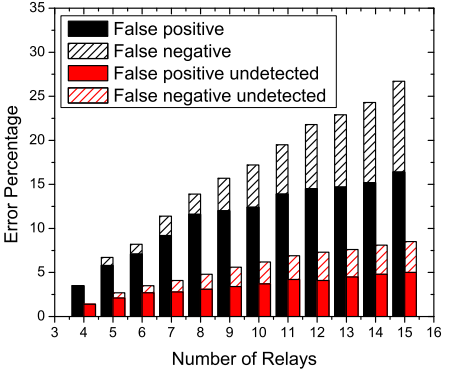

(a) Performance of error detection
Fig. 13. Performance of error detection in MIT Reality trace. The data lifetime is set as 1 week (168 hours).

the possible errors during redundancy elimination. Such CRI incompleteness, measured by the average size of relays' BZ, is shown in Figure 12 with Compare-and-Forward and CCP used for forwarding decision. Figure 12(a) shows that the average BZ size can be up to $35 \%$. Moreover, we also evaluate the effects of pre-regulation of forwarding process on reducing the average BZ size. The evaluation results in Figure 12(b) show that the average BZ size is reduced by up to $50 \%$ when $N_{R}=1$ but increases when either $N_{R}$ or $K_{R}$ increase.

The performance of error detection and correction schemes proposed in Section IV-C is evaluated in Figure 13. Figure 13(a) shows that majority of errors are false positive, and false negative errors are only noticeable when many message replicas are created. Our schemes can effectively detect both types of errors, and limit the cumulative error percentage lower than 10\%. Figure 13(b) also evaluates the delay of error detection. Obviously this delay is closely related with data lifetime. When data lifetime is set as 1 week for the MIT Reality trace, Figure 13(b) shows that both types of errors can be detected and corrected within $20 \%$ of the data lifetime.

Using the same setup, the schemes proposed in Section IV-C for improving the accuracy of redundancy elimination is evaluated in Figure 14. First, comparisons between Figure 10 and Figure 14 show that posterior adjustment of relays effectively corrects errors during redundancy elimination, and improves the forwarding performance to the level of global elimination. Second, Figure 14(b) shows that pre-regulation of forwarding process reduces forwarding redundancy by $10 \%$, but prevents some relays from receiving messages and reduces delivery ratio by $3 \%$. Hence, these two schemes have different 


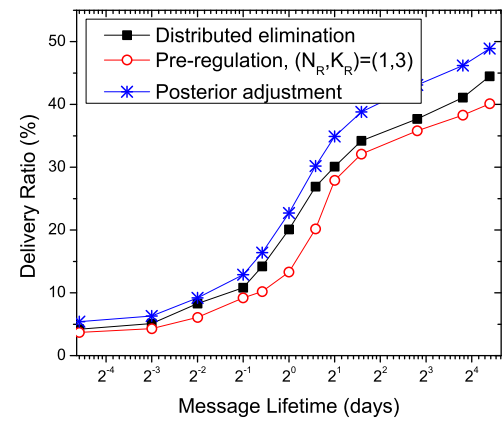

(a) Delivery ratio

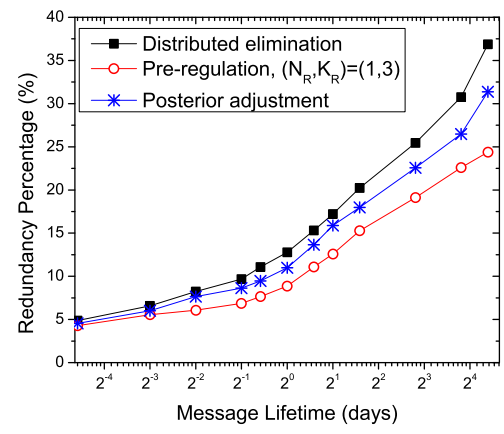

(b) Redundancy Percentage

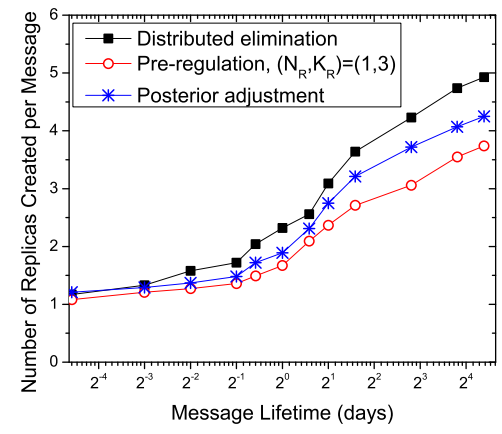

(c) Number of replicas created per message

Fig. 14. Performance of improving the accuracy of distributed redundancy elimination in the MIT Reality trace

tradeoff between forwarding performance and redundancy, and should be used according to the specific applications.

\section{RELATED WORK}

Research on forwarding in opportunistic mobile networks originates from Epidemic routing [19]. Later studies develop forwarding strategies to approach the performance of Epidemic with fewer relays. Representative strategies include Compare-and-Forward [3], [5], Delegation [6] and Spray-andFocus [18], which were exploited when studying forwarding redundancy in this paper. Utility functions evaluating nodes' contact capability are independent from strategies mentioned above. Some schemes predict node contact capability by estimating their co-location probabilities [20]. Some others [1], [10], [11] exploit node contacts as stochastic process for better prediction accuracy, based on experimental [14] and theoretical [2] analysis on contact characteristics.

Social properties of human mobility including centrality and community structures are also exploited for forwarding messages [13], [12]. BUBBLE Rap [13] considers node centrality hierarchically in social community structures. Gao et al. [12] exploits both properties for multicasting, and its proposed CCP utility function was also used in this paper.

\section{CONCLUSIONS}

In this paper, we studied forwarding redundancy in opportunistic mobile networks, which could impair the forwarding performance. We investigated its characteristics from both theoretical and experimental aspects, and proposed effective schemes to eliminate this redundancy with limited network information. Intensive evaluations show that our schemes can improve forwarding performance with much lower cost.

\section{REFERENCES}

[1] A. Balasubramanian, B. Levine, and A. Venkataramani. DTN Routing As a Resource Allocation Problem. In Proceedings of SIGCOMM, 2007.

[2] H. Cai and D. Y. Eun. Crossing over the bounded domain: from exponential to power-law inter-meeting time in manet. Proc. MobiCom, pages 159-170, 2007.

[3] H. Dubois-Ferriere, M. Grossglauser, and M. Vetterli. Age matters: efficient route discovery in mobile ad hoc networks using encounter ages. Proc. MobiHoc, pages 257-266, 2003.

[4] N. Eagle and A. Pentland. Reality mining: sensing complex social systems. Personal and Ubiquitous Computing, 10(4):255-268, 2006.

[5] V. Erramilli, A. Chaintreau, M. Crovella, and C. Diot. Diversity of Forwarding Paths in Pocket Switched Networks. In Proceedings of IMC, pages 161-174. ACM, 2007.
[6] V. Erramilli, A. Chaintreau, M. Crovella, and C. Diot. Delegation Forwarding. Proc. MobiHoc, 2008.

[7] K. Fall. A Delay-Tolerant Network Architecture for Challenged Internets. Proc. SIGCOMM, pages 27-34, 2003.

[8] L. Freeman. A set of measures of centrality based on betweenness. Sociometry, 40(1):35-41, 1977.

[9] W. Gao and G. Cao. On Exploiting Transient Contact Patterns for Data Forwarding in Delay Tolerant Networks. In Proceedings of ICNP, 2010.

[10] W. Gao and G. Cao. User-centric data dissemination in disruption tolerant networks. In Proceedings of INFOCOM, 2011.

[11] W. Gao, G. Cao, A. Iyengar, and M. Srivatsa. Supporting cooperative caching in disruption tolerant networks. In Proceedings of ICDCS, 2011.

[12] W. Gao, Q. Li, B. Zhao, and G. Cao. Social-aware multicast in disruption-tolerant networks. IEEE/ACM Transactions on Networking, 20(5):1553-1566, 2012.

[13] P. Hui, J. Crowcroft, and E. Yoneki. Bubble rap: social-based forwarding in delay tolerant networks. Proc. MobiHoc, 2008.

[14] T. Karagiannis, J.-Y. Boudec, and M. Vojnovic. Power law and exponential decay of inter contact times between mobile devices. Proc. MobiCom, pages 183-194, 2007.

[15] A. Lindgren, A. Doria, and O. Schelen. Probabilistic routing in intermittently connected networks. ACM SIGMOBILE CCR, 7(3):1920, 2003.

[16] S. Martello and P. Toth. Knapsack problems: algorithms and computer implementations. John Wiley \& Sons, 1990.

[17] M. McNett and G. Voelker. Access and mobility of wireless PDA users. ACM SIGMOBILE CCR, 9(2):40-55, 2005.

[18] T. Spyropoulos, K. Psounis, and C. Raghavendra. Efficient routing in intermittently connected mobile networks: The multiple-copy case. IEEE/ACM Transactions on Networking, 16(1):77-90, 2008.

[19] A. Vahdat and D. Becker. Epidemic routing for partially connected ad hoc networks. Technical Report CS-200006, Duke University, 2000.

[20] Q. Yuan, I. Cardei, and J. Wu. Predict and relay: an efficient routing in disruption-tolerant networks. In Proc. MobiHoc, pages 95-104, 2009.

[21] J. Zhao and G. Cao. VADD: Vehicle-Assisted Data Delivery in Vehicular Ad Hoc Networks. Proc. INFOCOM, 2006.

\section{APPENDIX}

Lemma 1 is proved by induction over levels of MRT.

Step 1: if $R_{k}$ is at the first level, $k=1$ and $R_{1}$ is the source node. The lemma simply holds.

Step 2: Suppose the lemma holds for all the relays at the $j$-th level. Since $N_{R}=1$, all the relays at the $(j+1)$-th level receive message replica from the same parent $R^{(j)}$ at the $j$-th level. For $R_{k}$ at the $(j+1)$-th level, $\left.i\right)$ it knows all the relays at the upper levels from $R^{(j)}$ because $\left|\widetilde{\mathbb{B}}_{R^{(j)}}(t)\right|=0$, ii) it knows all the relays at the $(j+1)$-th level with smaller index because they also receive message replica from by $R^{(j)}$. The lemma hence also holds for $R_{k}$ at the $(j+1)$-th level.

This lemma is proved by combining Steps 1 and 2 . 